Table 1. Characteristics of the 71 subjects included in analyses

Diagnosis $^{*}$

\begin{tabular}{lcc} 
& \multicolumn{1}{c}{ Diagnosis $^{\star \star}$} \\
\hline Age in years, mean (SD) & gout $(\mathrm{n}=63)$ & no gout $(\mathrm{n}=8)$ \\
Male gender, N (\%) & $62(14)$ & $59(14)$ \\
Symptom duration* at baseline in months, median (IQR) & $53(84)$ & $5(63)$ \\
Joint involvement at baseline N patients (\%): & $12(1-48)$ & $8(0.5-33)$ \\
MTP, & & \\
ankle/midfoot & $33(52)$ & $1(12)$ \\
other joint & $12(19)$ & $1(12)$ \\
SUA intercritical in umol/l, mean (SD) & $18(29)$ & $6(76)$ \\
2015 ACR/EULAR criteria baseline score, mean (SD) $)^{\star \star \star}$ & $484(63)$ & $337(71)$ \\
2015 ACR/EULAR criteria $\geq 8$ points, N patients (\%)*** & $10.3(2.5)$ & $2.6(1.5)$ \\
MSU crystal positive joint aspiration, N patients (\%) & $57(90)$ & $0(0)$ \\
DECT positive, N patients (\%) & $44(70)$ & $0(0)$ \\
\end{tabular}

* self-reported, intermittent symptoms; ** all patients classified with gout at baseline also had a clinical gout diagnosis after one yea; ${ }^{* \star *}$ using a somewhat limited set, see methods MTP, metatarsophalangeal joints; SUA, serum uric acid; DECT, dual-energy CT; MSU, monosodium urate;

Sensitivity, specificity, positive and negative predictive value, and accuracy values $(95 \% \mathrm{Cl})$ of the classification criteria set we used were $0.91(0.80-0.96) ; 1$ $(0.63-1) ; 1 ; 0.57(0.38-0.74)$ and $0.92(0.83-0.97)$, respectively. The area under the receiver operating characteristics curve $(95 \% \mathrm{Cl})$ was $0.95(0.91-0.99)$. ULT was started in $49 / 63(78 \%)$ of gout patients; $45 / 49(92 \%)$ of them had serum uric acid levels $\leq 360 \mu \mathrm{mol} / \mathrm{l}$ and no recurrent gout attack during one-year follow-up.

Conclusion: The 2015 ACR-EULAR gout classification criteria performed well for the diagnosis gout in clinical practice. Most gout patients had been treated successfully, according to current guidelines.

References:

[1] Gamala M, Jacobs JWG, Linn-Rasker SF, Nix M, Heggelman BGF, Pasker-de Jong PCM, et al. The performance of dual-energy CT in the classification criteria of gout: a prospective study in subjects with unclassified arthritis. Rheumatology 2019 Sep (Epub ahead print).

Disclosure of Interests: Mihaela Gamala: None declared, Johannes W. G. Jacobs Grant/research support from: Roche, Suzanne Linn-Rasker: None declared, Maarten Nix: None declared, Ben Heggelman: None declared, Pieternel Pasker: None declared, Jacob M. van Laar Grant/research support from: MSD, Genentech, Consultant of: MSD, Roche, Pfizer, Eli Lilly, BMS, Ruth Klaasen: None declared

DOI: 10.1136/annrheumdis-2020-eular.2457

\section{AB0927 HIGHER ULTRASOUND BURDEN WITH MONOSODIUM URATE CRYSTALS IN THE JOINTS IS CONNECTED TO MORE PRONOUNCED ARTERIOSCLEROTIC VASCULAR ALTERATIONS}

R. Gancheva ${ }^{1}$, T. Kundurzhiev ${ }^{2}$, Z. Kolarov ${ }^{1}$, A. Koundurdjiev ${ }^{3} .{ }^{1}$ University Hospital St. Ivan Rilski, Medical University, Clinic of Rheumatology, Sofia, Bulgaria; ${ }^{2}$ Medical University, Faculty of Public Health, Sofia, Bulgaria; ${ }^{3}$ University Hospital St. Ivan Rilski, Medical University, Clinic of Nephrology, Sofia, Bulgaria

Background: The severity of gout and the presence of subcutaneous tophi increase the risk of cardiovascular death. In patients (pts) in the spectrum of gout and in subjects who have inflammatory arthritis with accompanying asymptomatic hyperuricemia, data whether ultrasound (US) burden with monosodium urate (MSU) crystals in the joints is associated with higher cardiovascular risk are contradictory.

Objectives: To establish the relationship between US MSU crystal deposits in the joints with diastolic heart function and carotid arteries alterations in gout pts, individuals with asymptomatic hyperuricemia and no sign of inflammatory arthritis and psoriatic arthritis pts with asymptomatic hyperuricemia.

Methods: The study is cross-sectional. A total of 121 consecutive pts were included, divided into 85 pts with gout -63 males and 22 females aged $57.7 \pm 14.1$ years, 27 subjects with asymptomatic hyperuricemia and no sign of inflammatory arthritis - 13 males and 14 females aged $53.4 \pm 17.2$ years, and 9 psoriatic arthritis individuals with asymptomatic hyperuricemia - 2 males and 7 females aged $61.2 \pm 14.4$ years. Pts underwent two-dimensional echocardiography, US examination of the common carotid arteries and US of the joints of the hands, elbows, knees, ankles and feet. The echocardiography was conducted with 2.5 $\mathrm{MHz}$ transducer phased array working with pulse Doppler frequency of $2.5 \mathrm{MHz}$. Parameters of the transmitral blood flow were measured: the ratio between maximal early and late flow velocities (E/A ratio) and deceleration time (DT). With tissue Doppler imaging, early diastolic mitral annular velocity ( $\left.\mathrm{e}^{\prime}\right)$ was obtained. We judged for left ventricular filling pressure by determining $\mathrm{E} / \mathrm{e}^{\prime}$ ratio. Using US of the common carotid arteries done with $10 \mathrm{MHz}$ linear transducer working with pulse Doppler frequency of $5 \mathrm{MHz}$ were measured: intima-media thickness (IMT), common carotid artery resistive index (CCARI) and the presence of atherosclerotic plaques was recorded. US of the joints was performed with a high-frequency, linear transducer, 4-15 MHz. The existence of double contour sign, intra-tendinous MSU aggregates, snow storm, tophi, tophi with erosions, or a combination of these US features was assessed. Data were analyzed by ChiSquare, Mann-Whitney, Kruskal Wallis, t-test and ANOVA.

Results: In the three groups there was no difference in the mean values of E/A ratio $(p=0.591)$, DT $(p=0.498), e^{\prime}(p=0.662)$, E/e ratio $(p=0.754)$, IMT $(p=0.260)$ CCARI $(p=0.089)$ and in the frequency of heterogeneous or homogeneous carotid arteries plaques $(p=0.595)$. Among pts with and without evidence of MSU crystals in the joints the means of $E / A$ ratio $(p=0.452)$, DT $(p=0.367)$, e $(p=0.218)$, E/e ratio $(p=0.230)$, IMT $(p=0.165)$, CCARI $(p=0.097)$ and the frequency of heterogeneous or homogeneous plaques $(p=0.830)$ were comparable. The distribution of MSU crystal deposits in two or more joints was the highest in gout pts $(56.5 \%)$ compared to pts with asymptomatic hyperuricemia $(11.1 \%)$ and individuals with psoriatic arthritis $(22.2 \%),(p<0.001)$. Pts with MSU crystal deposits in two or more joints compared to those with crystal deposits in one join and pts without MSU crystals had the highest CCARI (mean \pm SD; $0.72 \pm 0.05$ vs $0.69 \pm 0.07$ vs $0.68 \pm 0.07, p=0.019$ ), the longest DT (mean $\pm S D ; 236 \pm 50.99 \mathrm{msec}$ vs $208.09 \pm 34.30 \mathrm{msec}$ vs $216 \pm 55.43 \mathrm{msec}, \mathrm{p}=0.026$ ) and had a tendency of lowest $e^{\prime}$ (mean $\pm S D ; 10.33 \pm 3.96 \mathrm{~cm} / \mathrm{s}$ vs $12.03 \pm 3.83 \mathrm{~cm} / \mathrm{s}$ vs $11.99 \pm 3.98 \mathrm{~cm} / \mathrm{s}$, $p=0.077)$, but the values of $E / A$ ratio $(p=0.119)$, $E / e^{\prime}$ ratio $(p=0.107)$, IMT $(p=0.151)$ and the distribution of atherosclerotic plaques $(p=0.920)$ were equal. Conclusion: Pts with higher US MSU burden have more pronounced left ventricular diastolic dysfunction and greater vascular stiffness. The changes in their vessels are mainly of the arteriosclerotic type.

Disclosure of Interests: None declared

DOI: 10.1136/annrheumdis-2020-eular.1328

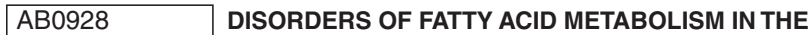 FORMATION OF ARTERIAL HYPERTENSION IN PATIENTS WITH GOUT}

M. Gromova ${ }^{1}$, V. Tsurko ${ }^{2}$, O. Kisliak ${ }^{1}$, E. Kiseleva ${ }^{3} .{ }^{1}$ Pirogov Russian National Research Medical University, Moscow, Russian Federation; ${ }^{2}$ I.M. Sechenov First Moscow State Medical University (Sechenov University), Moscow, Russian Federation; ${ }^{3}$ N.N. Semenov Federal Research Center for Chemical Physics, Russian Academy of Science, Moscow, Russian Federation

Background: One of the possible mechanisms of the formation of cardiovascular disorders in patients with gout may be a violation of the metabolism of fatty acids. Decrease of the total content of unsaturated fatty acids (UFAs) has a multifaceted adverse effect on a number of metabolic processes which lead to the development of arterial hypertension $(\mathrm{AH})$ and atherosclerosis as a result. There are no studies on the level of UFAs in the blood of patients with gout depending on the presence of hypertension.

Objectives: To study the level of UFAs in the blood of patients with gout depending on the presence of $\mathrm{AH}$.

Methods: We examined 87 patients with gout and $\mathrm{AH}$. $83 \%$ were men (mean age $55.4 \pm 12.3$ years). All patients had chronic gouty arthritis, $30 \%$ of patients had tofus. The duration of gout was $8[4 ; 11]$ years. $\mathrm{AH}$ was detected in 49 $(56.2 \%)$ people. The duration of $\mathrm{AH}$ was 7 [2; 10] years. All patients went through standard general clinical, laboratory and instrumental examination. Ozonation method was developed and put into practice in N.N. Semenov Federal Research Center for Chemical Physics, Russian Academy of Science (FRCCP RAS), to assess disorders of the lipid metabolism for diagnostic purposes. The essence of the method is to determine the level of unsaturation of serum or blood plasma lipids which depends on the total amount of double bonds (DB) in UFAs both in a free state and within lipid combination. This parameter is called the Double Bond Index (DBI). DBI is measured by a domestic device ADS-5M («Double bond analyzer») developed at FRCCP RAS. The control group consisted of 20 healthy men, comparable in age. Statistical analysis of the data was carried out using the STATISTICA 10.0 program.

Results: Patients were divided into 2 groups: patients with gout with normal blood pressure (group 1; $\mathrm{n}=38$ ) and with $\mathrm{AH}$ (group 2; $\mathrm{n}=49$ ). Patients from group 2 differed by a longer course of gout and had a higher level of uric acid in comparison with patients from group 1. The number of joints involved in the inflammatory process in patients from group 2 exceeded the number of those in patients from group 1. Patients from group 2 had more often attacks of gouty arthritis and the severity of pain during the last year compared with group 1. The normative value of the blood serum lipids DBI was determined for the control group of healthy people $(260+20$ conventional units (c.u.)) by the ozonation method. A significant deviation of the $\mathrm{DBI}$ from the norm both increased and decreased is a sign of pathology. In all groups $\mathrm{DBI}$ is lower than the norm but to a different extent. $\mathrm{DBI}$ is slightly reduced relative to the norm $(D B=229 \mathrm{c} . u$. $)$ in group 1 therefore lipid metabolism is impaired just slightly. DB = $167 \mathrm{c}$.u. in group 2 which indicates a significant disorder of lipid metabolism $(p<0,05)$. Thus the ozonation method is informative and allows 
to quantify the severity of patient general condition and $\mathrm{DBI}$ is an integral indicator of changes in the number of fatty acids and body general condition.

Conclusion: The presence of $\mathrm{AH}$ in patients with gout makes the clinical course of the disease more severe and exacerbates lipid disorders that can make significant changes in the formation of cardiovascular complications in this category of patients. DBI can be used as an additional criteria in laboratory diagnostics and monitoring to develop adequate treatment tactics.

Disclosure of Interests: Margarita Gromova Speakers bureau: Speaker for Biotehnos, Boehringer-ingelheim, Vladimir Tsurko Speakers bureau: Speaker for Boehringer-ingelheim, Berlin-chemie, Oksana Kisliak Speakers bureau: Speaker for Berlin-chemie, KRKA, Elena Kiseleva: None declared

DOI: 10.1136/annrheumdis-2020-eular.2213

\section{AB0929 CALCIUM PYROPHOSPHATE DIHYDRATE CRYSTAL DEPOSITS: REMEMBER THE SPINE!}

D. Khalifa ${ }^{1}$, K. Baccouche ${ }^{1}$, N. El Amri ${ }^{1}$, H. Zeglaoui ${ }^{1}$, E. Bouajina ${ }^{1} .{ }^{1}$ Farhat Hached Hospital, Rheumatology Department, Susah, Tunisia

Background: Calcium pyrophosphate dihydrate crystal-deposits (CPPD) is a common crystal disease affecting men and women equally. It is normally seen in peripheral joints. Spine involvement is rare and may mislead the diagnosis. Objectives: To describe clinical, radiological and therapeutic findings of CPPD of the spine.

Methods: A retrospective descriptive study was conducted in the rheumatology department of Farhat Hached Hospital, including patients diagnosed with CPPD of the spine over a period of 20 years (1998-2018). Data concerning clinical, radiological and therapeutic aspects of CPPD of the spine were callected from their medical files.

Results: Twelve patients had a spinal localisation of CPPD. The mean age was $59.91 \pm 16.21$ years. Patients were mainly women with a sex ratio men/women of 1:3. Mean duration of symptoms before the diagnosis was $27.08 \pm 25.69$ months. Spinal presentation was the revealing symptom of CPPD in $45.5 \%$ of the cases. It affected the cervical spine in $66.7 \%$ and the lumbar spine in $33.3 \%$ of the cases. Spinal localisation of CPPD was associated with a peripheral arthropatyhy in $75 \%$ of the cases (the knees in $41.7 \%$, wrists and hands in $66.6 \%$, shoulders in $25 \%$ and the hips in $16.7 \%$ ). Discovery of spinal CPPD was coincidental on plain radiographs in $16.7 \%$ of the cases. When symptomatic, the disease manifested itself as inflammatory pain in $72.7 \%$, mechanical pain in $9.1 \%$ and both diurnal and nocturnal pain in $18.2 \%$ of the cases. Physical examination revealed stiffness of the spine in $58.3 \%$ of the patients. Fever was noted in $8.3 \%$ and deterioration of general condition with anorexia was reported in $25 \%$ of the patients. Neurological complications were represented by cervical myelopathy in $25 \%$, sciatica in $8.3 \%$ and a case of unilateral ulnar nerve neuropathy was reported. Laboratory tests revealed inflammation in $50 \%$ of the cases. However, crystals were present in synovial fluid in only $25 \%$ of the patients. Spinal CPPD was visible on plain radiographs in $83.3 \%$ of the cases, showing calcified discs in $75 \%$ and signs of destruction in $16.7 \%$ of the cases. Peripheral calcifications were present in the wrists in $33.3 \%$ and the knees in $58.3 \%$ of the cases. Crow dens syndrome was reported in $16.7 \%$ of the patients. CT-scan helped the diagnosis of calcifications and crown dens syndrome when performed. MRI was prescribed in $58.3 \%$ of the patients and characterized the cervical myelopathy. Treatment relied on analgesics in all patients, NSAIDS were prescribed in $83.3 \%$, colchicine in $66.7 \%$ and general corticosteroids in $25 \%$ of the cases. Spinal immobilization was recommended in $25 \%$ of the cases for a short period. Spinal CPPD was idiopathic in $75 \%$, secondary to hyperparathyroidism in $8.3 \%$ of the cases and familial cases were detected in $16.7 \%$ of the patients.

Conclusion: Axial CPPD is rare and is an under-recognized entity that should be considered in elderly patients with neck or back pain. It can involve the discs or ligaments. The crown dens syndrome is quite suggestive of the diagnosis. If spinal CPDD is suspected, colchicine therapy could be a good therapeutic test and would avoid unnecessary further tests.

References:

[1] Ferrone C, Andracco R, Cimmino MA. Calcium pyrophosphate deposition disease: clinical manifestations. Reumatismo. 19 janv 2012;63(4):246-52.

[2] Feydy A, Lioté F, Carlier R, Chevrot A, Drapé J-L. Cervical spine and crystal-associated diseases: imaging findings. Eur Radiol. févr 2006;16(2):459-68. Disclosure of Interests: None declared

DOI: 10.1136/annrheumdis-2020-eular.4762

\section{$\mathrm{AB} 0930$ URATE INDUCED PRIMING OF HUMAN MONOCYTES IS MEDIATED VIA THE TGF-B PATHWAY}

V. Klück ${ }^{1}$, L. Mies ${ }^{1}$, R. Bakker ${ }^{2}$, T. Crisan ${ }^{3}$, L. Joosten ${ }^{1,3} \cdot{ }^{1}$ Radboudumc, Internal Medicine, Nijmegen, Netherlands; ${ }^{2}$ Radboudumc, Rheumatology, Nijmegen,

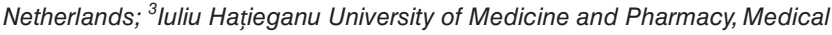
Genetics, Cluj Napoca, Romania
Background: Hyperuricemia, elevated serum urate levels, is the main risk factor for gout, but is also associated with higher incidence of comorbidities such as cardiovascular disease, type 2 diabetes, metabolic syndrome and chronic kidney disease[1]. Crisan et al. showed that urate leads to increased production of interleukin (IL)-1 $\beta$, a pro-inflammatory cytokine, and downregulation of IL-1 receptor antagonist (IL-1Ra), the natural inhibitor of IL-1, in human monocytes[2]. This imbalance between IL-1 $\beta$ and IL-1Ra is mediated by epigenetic reprogramming of innate immune cells[2]. RNA sequencing in urate-treated monocytes demonstrated that the TGF- $\beta$ signalling pathway was differentially expressed[3].

Objectives: The objective of this study is to further explore the role of TGF- $\beta$ in urate induced priming of human monocytes.

Methods: Human peripheral blood mononuclear cells (PBMCs) were isolated from healthy volunteers, adhered to a flat bottom plate, and treated for $24 \mathrm{~h}$ with a dosing range of urate after which mRNA was isolated. For validation experiments, PBMCs from 9 gout patients and 7 healthy controls were isolated and adhered to a flat bottom plate for $4 \mathrm{~h}$ after which cells were stored for RNA isolation. qPCR primers designed for TGF- $\beta$, TGF- $\beta$ receptor I and II, MMP9, SMAD7 and ITGAV were used to assess expression levels of TGF- $\beta$ pathway in these adherent monocytes. For priming experiments, adherent monocytes were primed for $24 \mathrm{~h}$ with urate and/or recombinant TGF- $\beta 1$ (R\&D systems) with or without a TGF- $\beta$ receptor II antibody (R\&D systems), cells were washed and restimulated with LPS for $24 \mathrm{~h}$. Cytokine levels in supernatant were determined by ELISA for IL-1 $1 \beta$, IL- 6 and IL-1Ra.

Results: mRNA expression of TGF- $\beta$ and its downstream targets were upregulated in urate treated monocytes and in gout patients compared to healthy controls. Moreover, urate levels significantly correlated to TGF- $\beta$ in individuals with gout. Both urate and TGF- $\beta$ priming increased the release of IL- $1 \beta$ and IL- 6 after LPS stimulation in human monocytes. We did not observe a synergistic effect between the two and therefore hypothesized that urate induced inflammation is mediated via TGF- $\beta$. Blocking the TGF- $\beta$ receptor II partly reversed the urate induced phenotype: lowered IL- $1 \beta$ and IL- 6 production and restored levels of $\mathrm{IL}-1 \mathrm{R}$. Further validation experiments are ongoing.

Conclusion: Urate induced priming of human monocytes is at least partly mediated via the TGF- $\beta$ pathway. This study contributes to the understanding of the pathways involved in urate induced inflammatory status and might in the future provide a mechanistic explanation for the occurrence of some comorbidities in patients with gout. Additionally, as TGF- $\beta$ is a major player in the pathogenesis of systemic sclerosis, this study might give a rationale for treatment of hyperuricemia in this population.

\section{References:}

[1] Bardin, T. and P. Richette, Impact of comorbidities on gout and hyperuricaemia: an update on prevalence and treatment options. BMC Med, 2017. 15(1): p. 123.

[2] Crisan, T.O., et al., Soluble uric acid primes TLR-induced proinflammatory cytokine production by human primary cells via inhibition of IL-1Ra. Ann Rheum Dis, 2016. 75(4): p. 755-62.

[3] Crisan, T.O., et al., Uric acid priming in human monocytes is driven by the AKT-PRAS40 autophagy pathway. Proc Natl Acad Sci U S A, 2017. 114(21): p. 5485-5490.

Disclosure of Interests: Viola Klück: None declared, Linda Mies: None declared René Bakker: None declared, Tania Crisan: None declared, Leo Joosten Consultant of: SAB member of Olatec Therapeutics LLC

DOI: 10.1136/annrheumdis-2020-eular.5548

\section{AB0931 THE QUALITY OF LIFE IN GOUT PATIENTS WITH ULCERATION OVER TOPHI}

X. Liu ${ }^{1,2}$, Z. Huang ${ }^{1}$, Q. Huang ${ }^{1}$, Z. Zhong ${ }^{1,2}$, W. Zhao ${ }^{1,3}$, T. LI $^{1,2} .{ }^{1}$ Guangdong Second Provincial General Hospital, Department of Rheumatology and Immunology, Guangzhou; ${ }^{2}$ Southern Medical University, The Second School of Clinical Medicine, Guangzhou, China; ${ }^{3}$ University of South China, Hengyang, China

Background: The prevalence of gouty patients with ulcerations over tophi are increasing over time and it has been reported that gouty patients have significantly poor quality of life (QoL) compared to those healthy controls. [1, 2] there is no study on comparison of the QoL in patients with or without ulceration over tophi.

Objectives: To compare the QoL in gout patients with or without ulcerations over tophi.

Methods: A total of 79 inpatients with gout who were admitted to Guangdong Second Provincial General Hospital from January 2019 to January 2020 were included. Among them, ulcerations were identified in 28 patients. Short Form-36 Scales (SF-36) were chosen to assess patients. Differences between the groups were tested with Student's t test or Mann-Whitney $U$ test for continuous variables, and Chi-square tests for categorical variables.

Results: Of 79 subjects, 74 were male, mean age was $54.23 \pm 14.3$ years, disease duration was $10.94 \pm 7.06$ years. Gouty patients had moderate- to high-quality levels in general health, vitality, social functioning, role emotional, and 